\title{
Effect of Macromolecular Polydispersity on Diffusion Coefficients Measured by Rayleigh Interferometry
}

\author{
Huixiang Zhang ${ }^{\dagger, \dagger}$ and Onofrio Annunziata ${ }^{*, \dagger}$ \\ Department of Chemistry, Texas Christian University, Fort Worth, Texas 76129, and Alcon Research Ltd., \\ Fort Worth, Texas 76134
}

Received: October 29, 2007; In Final Form: December 17, 2007

\begin{abstract}
Rayleigh interferometry has been extensively used for the precise determination of diffusion coefficients for binary and ternary liquid mixtures. For ternary mixtures, the $2 \times 2$ matrix of multicomponent diffusion coefficients is obtained. Polydispersity adds complexity to the meaning of these measured diffusion coefficients. Here we discuss three important issues of polydispersity regarding the diffusion measurements extracted from this interferometric technique. First, we report novel equations for the extraction of diffusion moments from the Rayleigh interferometric pattern. These moments are used to define polydispersity parameters for macromolecular systems. We have experimentally determined mean diffusion coefficients and polydispersity parameters for aqueous solutions of poly(ethylene glycol) and poly(vinyl alcohol) at $25^{\circ} \mathrm{C}$. Aqueous solutions of poly(ethylene glycol) mixtures were used to examine the accuracy of the polydispersity parameters. Second, we compare Rayleigh interferometry to dynamic light scattering. Specifically, we have performed diffusion measurements on the same system using both techniques. To our knowledge, no direct experimental comparison between dynamic light scattering and classical methods for the measurements of diffusion coefficients has been previously reported in relation to polydispersity. We find that substantial discrepancies (i.e., 1 order of magnitude) between the mean diffusion coefficients obtained from these two different techniques can be observed when polydispersity is large. Third, for two-solute mixtures with one polydisperse solute, we report a novel corrective procedure for extracting accurate ternary diffusion coefficients from Rayleigh interferometry. Computer simulations were used to examine the accuracy of the extracted ternary diffusion coefficients.
\end{abstract}

\section{Introduction}

Diffusion coefficients of mixtures containing large solutes $(1-100 \mathrm{~nm})$ such as polymers, proteins, micelles, vesicles, and other nanoparticles are important for a large number of laboratory, biological, and industrial processes. They have been measured for two main reasons: (1) they are fundamental physicochemical quantities related to particle size, aggregation, and molecular interactions in solution; (2) they are necessary parameters for modeling, predicting and designing the dynamic behavior of these processes. ${ }^{1-3}$ One important property of macromolecules and colloidal particles is polydispersity. Since diffusion coefficients are very sensitive parameters to particle size, diffusion measurements in dilute solutions yield the moments of the particle-size distribution. ${ }^{4-11}$

Currently, dynamic light scattering $(\mathrm{DLS})^{7}$ is the most commonly used technique for measuring diffusion coefficients of macromolecules and colloidal particles. In this case, mutual diffusion coefficients are obtained from relaxation times of microscopic concentration fluctuations in solution. Classical techniques such as Rayleigh interferometry, ${ }^{13,14}$ Gouy interferometry, ${ }^{15,16}$ and Taylor dispersion ${ }^{17}$ have been also used for diffusion measurements in macromolecular solutions. In these other cases, mutual diffusion coefficients are extracted from the spatial evolution of macroscopic concentration gradients designed by the experimentalist. For polydisperse solutes, the type

* To whom correspondence should be addressed: Phone: (817) 2576215. Fax: (817) 257-5851. E-mail: O.Annunziata@tcu.edu.

$\uparrow$ Texas Christian University.

$\doteqdot$ Alcon Research Ltd. of moments of particle-size distributions depends on the technique employed to measure diffusion coefficients. Thus, DLS moments are different from those obtained using classical techniques.

Contrary to DLS, classical techniques can be also used to investigate multicomponent diffusion; ${ }^{17-19}$ i.e., the determination of the complete diffusion-coefficient matrix for systems containing more than one solute. For instance, the $2 \times 2$ matrix of four diffusion coefficients has been reported for several ternary systems containing a macromolecular solute. ${ }^{19-29}$ The Gosting diffusiometer operating in the Rayleigh optical configuration has generated the most accurate multicomponent diffusion data. ${ }^{19-23}$ However, polydispersity of a macromolecular solute adds complexity to the interpretation of the diffusion data. This has been recently addressed by Mangiapia et al. ${ }^{10}$ For solutions containing one polydisperse solute, their paper reports a procedure for extracting meaningful and reliable multicomponent diffusion coefficients using Gouy interferometry.

Here we discuss several aspects of polydispersity relevant to Rayleigh interferometry. We report measurements of diffusion moments by Rayleigh interferometry on macromolecular systems. Specifically, we have determined a mean diffusion coefficient and polydispersity indices for aqueous solutions of poly(ethylene glycol) and poly(vinyl alcohol) at $25^{\circ} \mathrm{C}$. We have then performed DLS measurements on the same polydisperse systems and compared the results of these two techniques. To our knowledge, no direct experimental comparison between DLS and classical techniques has been previously reported in relation to polydispersity. Finally, for ternary mixtures containing one 
polydisperse solute, we report a novel procedure for extracting reliable multicomponent diffusion coefficients using Rayleigh interferometry.

Rayleigh interferometry provides the one-dimensional profile of refractive index inside a vertical channel filled with liquid. ${ }^{30}$ Diffusion inside the channel is brought about by an initial sharp boundary between two solutions with different composition and, consequently, different refractive index. The refractive-index profile inside the channel is described by the function $f=2(n$ $-\bar{n}) / \Delta n$ where $n$ is the refractive index at a given position, $x$, inside the channel, and $\bar{n}$ and $\Delta n$ are respectively the average and the difference in refractive index between the two solutions. The function $f$ is obtained by locating the fringe position of the resulting Rayleigh interference pattern. Multicomponent diffusion coefficients are normally obtained by applying a nonlinear least-squares method to $f$ within the free-diffusion boundary condition. Sundelöf ${ }^{4}$ has theoretically shown for polydispersity that the derivatives $\partial^{i} f / \partial x^{i}$ (with $i=1,2,3, \ldots$ ) extrapolated to the boundary position, $x=0$, can be used to extract a corresponding set of moments of the particle-size distribution. Clearly, these derivatives are associated with a power-series expansion of $f$ with respect to $x=0$. Here, on the other hand, we will show how to extract the same moments from the corresponding power-series expansion of $\operatorname{erfinv}(f)$. This different choice is motivated by its better convergence property. ${ }^{31}$

\section{Theory}

Effect of Macromolecular Polydispersity on Diffusion Coefficients of Macromolecule-Solvent Systems. In this section, we will first outline the well-established diffusion equations used for Rayleigh interferometry. We will then theoretically show how to extract the moments of the particlesize distribution from the power-series expansion of $\operatorname{erfinv}(f)$.

Diffusion Equations. For a system with $N$ macromolecular components and a solvent, generalized Fick's first law is ${ }^{2}$

$$
-J_{i}=\sum_{j=1}^{N} D_{i j} \nabla C_{j} \quad \text { with } i=1, \ldots, N
$$

In eq $1, J_{i}$ is the flux of component $i, C_{j}$ is the concentration of component $j$, and $D_{i j}$ is the diffusion coefficient that relates the flux of $i$ to the concentration gradient of $j$. For convenience, we will define $J_{i}$ and $C_{j}$ with respect to the mass of the macromolecular components.

If the $D_{i j}$ values are constant and $n$ linearly depends on $C_{j}$, the refractive-index profile associated with the free-diffusion boundary condition is described by the linear combination of $N$ error functions: ${ }^{3,10}$

$$
f=\sum_{k=1}^{N} \Gamma_{k} \operatorname{erf}\left(y / \sqrt{\Lambda_{k}}\right)
$$

where

$$
\Gamma_{k} \equiv \sum_{i=1}^{N} \sum_{j=1}^{N}\left(R_{i} / R_{j}\right) T_{i k} T_{k j}^{-1} \alpha_{j}
$$

In eq $2, y \equiv x / 2 \sqrt{t}$, where the spatial position $x=0$ and time $t=0$ corresponds to the initial sharp boundary between the two solutions. In eq $3, \alpha_{i} \equiv R_{i} \Delta C_{i} / \Delta n, R_{i} \equiv\left(\partial n / \partial c_{i}\right), \Delta c_{i}$ is the difference in $i$ concentration between the two initial solutions, and $\sum_{k=1}^{N} \Gamma_{k}=1$. The $\Lambda_{k}$ values are the eigenvalues of the diffusion-coefficient matrix, while $T_{i k}$ and $T_{i k}{ }^{-1}$ are the elements of the eigenvector matrix and its inverse, respectively. The function $f$ in eq 2 is antisymmetric with respect to $y=0$. However, the $D_{i j}$ values are generally not constant, and, consequently, $f$ may not be antisymmetric. To avoid this problem, Creeth's antisymmetrization procedure (Creeth-pair method) has been normally applied, yielding a corrected $f(y)$, which is defined only for $y \geq 0$. This function is normally used for the determination of multicomponent diffusion coefficients.

For dilute solutions, cross-diffusion coefficients can be neglected, and eq 2 becomes ${ }^{31,32}$

$$
f=\sum_{k} w_{k} \operatorname{erf}\left(y / \sqrt{D_{k}}\right)
$$

where $D_{k} \equiv D_{k k}=\Lambda_{k}$, and $w_{k}=\Gamma_{k}$ is the weight-fraction contribution of species $f$ to the macromolecule total mass. In eq 3 , we have also assumed that the $R_{i}$ values are all equal to each other. This is a very good approximation for macromolecular components with different size but the same chemical composition.

Diffusion Moments. A linear combination of error functions can be formally rewritten as a single error function of a series expansion in $y$ with respect to $y=0$. Thus, eq 2 can be rewritten as

$$
f=\operatorname{erf}(s y)
$$

where

$$
s=\sum_{i=0}^{\infty} a_{i} y^{2 i}
$$

where the $a_{i}$ are coefficients of the series expansion. For a monodisperse macromolecule with diffusion coefficient, $D$, we obtain $s=a_{0}=D^{-1 / 2}$ and $a_{i}=0$ for $i \neq 0$. For a polydisperse macromolecule, the function $s(y)$ can be calculated from $f(y)$ using eq 5 . Hence, the $a_{i}$ can be experimentally determined, thereby providing information on macromolecular polydispersity.

We will now show how to relate the $a_{i}$ to the diffusion moments relative to the distribution of the $\Gamma_{k}$ 's. By using the mathematical identity $\operatorname{erf}(x)=(2 / \sqrt{\pi}) \sum_{i=0}^{\infty}\left\{(-1)^{i} x^{2 i+1}\right\} /\{(2 i+$ $1) i$ ! \}, we can write the following two equivalent results:

$$
\begin{aligned}
f=\operatorname{erf}\left(y \sum_{i=0}^{\infty} a_{i} y^{2 i}\right)= & \frac{2}{\sqrt{\pi}} \sum_{n=0}^{\infty} \frac{(-1)^{n} y^{2 n+1}}{(2 n+1) n !}\left(\sum_{i=0}^{\infty} a_{i} y^{2 i}\right) 2 n+1 \\
f=\sum_{k=1}^{N} \Gamma_{k} \operatorname{erf}\left(y \lambda_{k}^{-1 / 2}\right) & = \\
& \frac{2}{\sqrt{\pi}} \sum_{n=0}^{\infty} \frac{(-1)^{n} y^{2 n+1}}{(2 n+1) n !} \sum_{k=1}^{N} \Gamma_{k} \Lambda_{k}^{-(2 n+1) / 2}
\end{aligned}
$$

By equating eq 7 and eq 8 , we obtain the following identities:

$$
\begin{gathered}
\left\langle D^{-1 / 2}\right\rangle=a_{0} \\
\left\langle D^{-3 / 2}\right\rangle=a_{0}^{3}-3 a_{1} \\
\left\langle D^{-5 / 2}\right\rangle=a_{0}{ }^{5}-10 a_{0}{ }^{2} a_{1}+10 a_{2}
\end{gathered}
$$

where $\left\langle D^{-(2 n+1) / 2}\right\rangle \equiv \sum_{k=1}^{N} \Gamma_{k} \Lambda_{k}{ }^{-(2 n+1) / 2}$ with $n=0,1,2,3, \ldots$ For dilute solutions, we obtain $\left\langle D^{-(2 n+1) / 2}\right\rangle=\sum_{k=1}^{N} w_{k} D_{k}^{-(2 n+1) / 2}$; 
i.e., the $\left\langle D^{-(2 n+1) / 2}\right\rangle$ values become the diffusion moments of the weight-fraction distribution. The momentum of lowest order is the mean diffusion coefficient $D_{\mathrm{A}}$ :

$$
D_{\mathrm{A}} \equiv\left\langle D^{-1 / 2}\right\rangle^{-2}=a_{0}^{-2}
$$

Polydispersity can be quantified by considering the diffusion momenta of higher order. Hence, we define the following polydispersity indices:

$$
\begin{gathered}
\omega \equiv \frac{\left\langle D^{-3 / 2}\right\rangle}{\left\langle D^{-1 / 2}\right\rangle^{3}}-1=-3 \frac{a_{1}}{a_{0}^{3}} \\
\xi \equiv \frac{\left\langle D^{-5 / 2}\right\rangle}{\left\langle D^{-1 / 2}\right\rangle^{5}}-1=-10 \frac{a_{1}}{a_{0}^{3}}+10 \frac{a_{2}}{a_{0}^{5}}
\end{gathered}
$$

These can be determined from the experimentally determined $a_{i}$.

Molecular-Weight Polydispersity. All diffusion techniques provide only indirect information on the molecular-weight polydispersity. This is described by the ratios $\bar{M}_{\mathrm{w}} / \bar{M}_{\mathrm{n}}=$ $\sum_{k=1}^{N} w_{k} M_{k} \sum_{k=1}^{N} w_{k} / M_{k}$ and $\bar{M}_{z} / \bar{M}_{\mathrm{w}}=\sum_{k=1}^{N} w_{k} M_{k}^{2} /\left(\sum_{k=1}^{N} w_{k} M_{k}\right)^{2}$, where $M_{k}$ is the molecular weight of species $k$, and $\bar{M}_{\mathrm{n}}, \bar{M}_{\mathrm{w}}$ and $\bar{M}_{z}$ are the number-average, mass-average, and z-average molecular weights, respectively. ${ }^{33,34}$ These quantities are not directly related to $\omega$ and $\xi$.

A relation between molecular-weight polydispersity and diffusion parameters may be obtained if a given analytical form of the $w_{k}$ distribution ${ }^{34}$ is assumed. Here we report the important example of the generalized exponential distribution applied to polymers with molecular weight $M_{k}=k M_{1}$, where $M_{1}$ is the monomer molecular weight: ${ }^{34}$

$$
w_{k}=\frac{m l^{(q+1) / m}}{\Gamma[(h+1) / m]} k^{h} \mathrm{e}^{-l k^{m}}
$$

In eq 13 , the parameters $l, h$, and $m$ characterize the position, width, and shape of the distribution, respectively. The massaverage degree of polymerization, $\langle k\rangle$, is

$$
\langle k\rangle=\frac{\Gamma[(h+2) / m]}{l^{1 / m} \Gamma[(h+1) / m]}
$$

where we have used the definition $\left\langle k^{n}\right\rangle \equiv \sum_{k=1}^{N} w_{k} k^{n}$ with $n$ being any real number, and $\Gamma$ is the gamma function. Equation 13 is a generalization of the Schulz-Zimm distribution, ${ }^{34}$ which is obtained by setting $m=1$. The Flory most probable distribution is obtained by setting $m=h=1$. Finally, we also observe that the Schulz-Zimm distribution approximates the Poisson distribution ${ }^{34}$ if $m=l=1$, i.e., $h=\langle k\rangle$.

For polymers in dilute solutions, $D_{k} \propto R_{\mathrm{h} k}{ }^{-1} \propto M_{k}{ }^{-a},{ }^{8}$ where $R_{\mathrm{h} k}$ is the hydrodynamic radius of species $k$, and $a$ is a scaling coefficient. We observe that $a=1 / 2$ for random coils, and $a=$ $1 / 3$ for compact spherical particles. Since $D_{k} \propto k^{-a}$, it can be shown that ${ }^{34}$

$$
\begin{gathered}
\bar{M}_{w} / \bar{M}_{n}=\frac{\Gamma[(h+2) / m] \Gamma(h / m)}{\Gamma[(h+1) / m]^{2}} \\
\bar{M}_{z} / \bar{M}_{w}=\frac{\Gamma[(h+3) / m] \Gamma[(h+1) / m]}{\Gamma[(h+2) / m]^{2}}
\end{gathered}
$$

Similar expressions can be obtained for $\omega$ and $\xi$ :

$$
\begin{aligned}
& \omega=\frac{\left\langle M^{(3 / 2) a}\right\rangle}{\left\langle M^{(1 / 2) a}\right\rangle^{3}}-1= \\
& \frac{\Gamma\{[h+(3 / 2) a+1] / m\} \Gamma\{(h+1) / m\}^{2}}{\Gamma\{[h+(1 / 2) a+1] / m\}^{3}}-1
\end{aligned}
$$

$$
\begin{aligned}
\xi=\frac{\left\langle M^{(5 / 2) a}\right\rangle}{\left\langle M^{(1 / 2) a}\right\rangle^{5}}-1= \\
\frac{\Gamma\{[h+(5 / 2) a+1] / m\} \Gamma\{(h+1) / m\}^{4}}{\Gamma\{[h+(1 / 2) a+1] / m\}^{5}}-1
\end{aligned}
$$

Equations $15-18$ relate $\omega$ and $\xi$ to $\bar{M}_{\mathrm{w}} / \bar{M}_{\mathrm{n}}$ and $\bar{M}_{z} / \bar{M}_{\mathrm{w}}$ through the coefficients $h$ and $m$.

Effect of Macromolecular Polydispersity on Ternary Diffusion Coefficients of Macromolecule-Additive-Solvent Systems. Monodisperse Macromolecular Solute. Rayleigh interferometry has been used to obtain ternary diffusion coefficients for macromolecule $(p)$-additive $(s)$-solvent ternary systems. However, these investigations have been limited to monodisperse macromolecules (i.e., lysozyme). ${ }^{19-23}$ Here, a generalized form of Fick's first law is

$$
\begin{aligned}
& -J_{p}=D_{p p} \nabla C_{p}+D_{\mathrm{ps}} \nabla C_{s} \\
& -J_{s}=D_{s p} \nabla C_{p}+D_{s s} \nabla C_{s}
\end{aligned}
$$

Main-term diffusion coefficients, $D_{p p}$ and $D_{s s}$, describe the flux of a solute due to its own mass concentration gradient, while cross-term diffusion coefficients, $D_{p s}$ and $D_{s p}$, describe the flux of a solute due to the mass concentration gradient of the other solute. The four ternary diffusion coefficients have been used for determining macromolecule-additive thermodynamic interactions (preferential-interaction coefficients) ${ }^{20-24}$ and for accurate modeling of diffusion processes..$^{35,36}$

The four diffusion coefficients are obtained by performing at least two experiments with different initial conditions. Normally, four experiments are performed to minimize experimental errors. A given initial condition can be described by $\alpha_{p}$ $=R_{p} \Delta C_{p} / \Delta n$ or $\alpha_{s}=R_{s} \Delta C_{s} / \Delta n=1-\alpha_{p}$, where $\Delta C_{p}$ and $\Delta C_{s}$ are the difference in macromolecule and additive concentrations between the two solutions. The two most employed initial conditions correspond to $\alpha_{p}=0$ and $\alpha_{p}=1$. For a generic $\alpha_{p}$, the experimental $f\left(y, \alpha_{p}\right)$ can be written in the following way:

$$
\begin{array}{r}
f=\left[\Gamma_{p}{ }^{\left(\alpha_{p}=0\right)}+\left(\Gamma_{p}{ }^{\left(\alpha_{p}=1\right)}-\Gamma_{p}{ }^{\left(\alpha_{p}=0\right)}\right) \alpha_{p}\right] \operatorname{erf}\left(y / \sqrt{\Lambda_{p}}\right)+[1- \\
\left.\Gamma_{p}{ }^{\left(\alpha_{p}=0\right)}-\left(\Gamma_{p}{ }^{\left(\alpha_{p}=1\right)}-\Gamma_{p}{ }^{\left(\alpha_{p}=0\right)}\right) \alpha_{p}\right] \operatorname{erf}\left(y / \sqrt{\Lambda_{s}}\right)
\end{array}
$$

where

$$
\begin{aligned}
\Gamma_{p}{ }^{\left(\alpha_{p}=0\right)} & =\frac{|D| \Lambda_{p}^{-1}-D_{s s}-\left(R_{p} / R_{s}\right) D_{p s}}{|D|\left(\Lambda_{p}^{-1}-\Lambda_{s}^{-1}\right)} \\
\Gamma_{p}{ }^{\left(\alpha_{p}=1\right)} & =\frac{D_{p p}+\left(R_{s} / R_{p}\right) D_{s p}-|D| \Lambda_{p}{ }^{-1}}{|D|\left(\Lambda_{s}^{-1}-\Lambda_{p}^{-1}\right)}
\end{aligned}
$$

and $|D|=D_{p p} D_{s s}-D_{p s} D_{s p}$. The four diffusion coefficients can be calculated from the values of $\Gamma_{p}{ }^{\left(\alpha_{p}=0\right)}, \Gamma_{p}{ }^{\left(\alpha_{p}=1\right)}, \Lambda_{p}$, and $\Lambda_{s}$ extracted from the nonlinear least-square method $^{37}$ applied to eq 20 .

We now observe that the two cross-diffusion coefficients, $D_{p s}$ and $D_{s p}$, are mainly related to the parameters $\Gamma_{p}{ }^{\left(\alpha_{p}=0\right)}$ and $\Gamma_{p}^{\left(\alpha_{p}=1\right)}$, respectively. Since $\Lambda_{p} \approx D_{p p}, \Lambda_{s} \approx D_{s s}$, and $|D| \approx$ 
$D_{p p} D_{s s}$ for most investigated macromolecule-additive systems, eqs $21 \mathrm{a}, \mathrm{b}$ can be approximated by

$$
\begin{gathered}
\Gamma_{p}{ }^{\left(\alpha_{p}=0\right)} \approx-\frac{\left(R_{p} / R_{s}\right) D_{p s}}{D_{s s}-D_{p p}} \\
\Gamma_{p}{ }^{\left(\alpha_{p}=1\right)} \approx 1-\frac{\left(R_{s} / R_{p}\right) D_{s p}}{D_{s s}-D_{p p}}
\end{gathered}
$$

Equations 22a,b allow us to observe that the determination of $D_{p s}$ mainly relies on the experiment at $\alpha_{p}=0$, while the determination of $D_{s p}$ mainly relies on the experiment at $\alpha_{p}=1$.

Polydisperse Macromolecular Solute. For macromolecule$(p)$-additive $(s)$-solvent systems with a polydisperse macromolecule, generalized Fick's first law becomes

$$
\begin{gathered}
-J_{i}=\sum_{j=1}^{N} D_{i j} \nabla C_{j}+D_{i s} \nabla C_{s} \text { with } i=1, \ldots, N \\
-J_{s}=\sum_{j=1}^{N} D_{s j} \nabla C_{j}+D_{s s} \nabla C_{s}
\end{gathered}
$$

The refractive-index profile associated with the free-diffusion boundary condition is described by

$$
f=\sum_{k=1}^{N} \Gamma_{k} \operatorname{erf}\left(y / \sqrt{\Lambda_{k}}\right)+\Gamma_{s} \operatorname{erf}\left(y / \sqrt{\Lambda_{s}}\right)
$$

where

$$
\begin{array}{r}
\Gamma_{k} \equiv \sum_{i=1}^{N} \sum_{j=1}^{N}\left(R_{i} / R_{j}\right)\left(T_{i k} T_{k j}{ }^{-1}\right) \alpha_{j}+\sum_{i=1}^{N}\left(R_{i} / R_{s}\right)\left(T_{i k} T_{k s}^{-1}\right) \alpha_{s}+ \\
\sum_{i=1}^{N}\left(R_{s} / R_{i}\right)\left(T_{s k} T_{k i}^{-1}\right) \alpha_{i}+\left(T_{s k} T_{k s}^{-1}\right) \alpha_{s} \\
\Gamma_{s} \equiv \sum_{i=1}^{N} \sum_{j=1}^{N}\left(R_{i} / R_{j}\right)\left(T_{i s} T_{s j}^{-1}\right) \alpha_{j}+\sum_{i=1}^{N}\left(R_{i} / R_{s}\right)\left(T_{i s} T_{s s}^{-1}\right) \alpha_{s}+ \\
\sum_{i=1}^{N}\left(R_{s} / R_{i}\right)\left(T_{s s} T_{s i}^{-1}\right) \alpha_{i}+\left(T_{s s} T_{s s}^{-1}\right) \alpha_{s}
\end{array}
$$

with $\Gamma_{s}+\sum_{k=1}^{N} \Gamma_{k}=1$. The determination of the $(N+1)^{2}$ diffusion coefficients from eqs $24 \mathrm{a}, \mathrm{b}$ is not feasible not only because of the large number of parameters to experimentally determine them, but also because the $\alpha_{j}$ values cannot be changed arbitrarily with respect to one another, contrary to $\alpha_{p}$ $=\sum_{i=1}^{\infty} \alpha_{i}$. However, we can analyze the experimental $f\left(y, \alpha_{p}\right)$ by considering an equivalent ternary system with a monodisperse macromolecular solute. ${ }^{10}$ The corresponding four ternary diffusion coefficients will be still valuable for modeling mixing processes and for the determination of macromolecule-additive interactions in the presence of mild polydispersity. We will now show how to approximately convert eq 24 into eq 20, provided that an appropriate correction is performed. The accuracy of the ternary diffusion coefficients determined using the proposed corrective procedure will then be examined using computer simulations.

Corrective Procedure. Our corrective procedure requires the introduction of reasonable approximations. They are all based on the observation that the chief effect of polydispersity on $f\left(y, \alpha_{p}\right)$ originates from the width of the spectrum of the $D_{i i}$ with $i=1,2,3, \ldots, N$. As for the macromolecule-solvent system, we shall set $R_{i}=R_{p}$ with $k=1,2,3, \ldots, N$, and neglect the crossdiffusion coefficients $D_{i j}$ with $i, j=1,2,3, \ldots, N$ and $i \neq j$. The latter approximation is excellent for dilute macromolecular solutions. We define the macromolecule main-diffusion coefficient, $D_{p p}$, of the equivalent ternary system by setting $D_{p p} \equiv$ $\left(\sum_{i=1}^{\infty} w_{i} D_{i i}{ }^{-1 / 2}\right)^{-2}$. This is consistent with the definition of mean diffusion coefficient shown by eq 10. The cross-diffusion coefficients $D_{p s}$ and $D_{s p}$ of the equivalent ternary system are introduced as follows. We will assume that all $D_{s j}$ 's are equal to each other and set as $D_{s j}=D_{s p}$. For $D_{p s}$, we observe that each $D_{i s}$ is proportional to the corresponding $C_{i}$. Thus, we set: $D_{i s}=w_{i} D_{p s}$.

We will now focus on the two initial conditions $\alpha_{p}=0$ and $\alpha_{p}=1$, for which eqs $25 \mathrm{a}, \mathrm{b}$ become

$$
\Gamma_{k}^{\left(\alpha_{p}=0\right)}=\left(R_{p} / R_{s}\right) \sum_{i=1}^{N}\left(T_{i k} T_{k s}^{-1}\right)+\left(T_{s k} T_{k s}^{-1}\right)
$$

$$
\begin{aligned}
\Gamma_{k}^{\left(\alpha_{p}=1\right)}= & \\
& \sum_{i=1}^{N} \sum_{j=1}^{N}\left(T_{i k} T_{k j}^{-1}\right) w_{j}+\left(R_{s} / R_{p}\right) \sum_{i=1}^{N}\left(T_{s k} T_{k i}^{-1}\right) w_{i}
\end{aligned}
$$

Here we will introduce important approximations that will be examined later by computer simulations. As previously observed, the experiments at $\alpha_{p}=0$ and $\alpha_{p}=1$ are not considerably dependent on the values of $D_{s p}$ and $D_{p s}$, respectively. Thus, we can set $D_{s p}=0$ for the specific experiment at $\alpha_{p}=0$, and $D_{p s}=0$ for the specific experiment at $\alpha_{p}=1$. Within this approximation, eqs 26 become

$$
\begin{gathered}
\Gamma_{k}^{\left(\alpha_{p}=0\right)} \approx w_{k} \frac{\left(R_{p} / R_{s}\right) D_{p s}}{D_{k k}-D_{s s}} \\
\Gamma_{k}^{\left(\alpha_{p}=1\right)} \approx w_{k}\left(1+\frac{\left(R_{s} / R_{p}\right) D_{s p}}{D_{k k}-D_{s s}}\right)
\end{gathered}
$$

The eigenvector matrices used for obtaining eqs $27 \mathrm{a}, \mathrm{b}$ from eqs $26 \mathrm{a}, \mathrm{b}$ are reported in the Appendix. For macromolecules, $D_{k k}$ is significantly smaller than $D_{s s}$. We can therefore write $D_{k k}-$ $D_{s s} \approx D_{p p}-D_{s s}$. This approximation allows us to directly compare eqs $27 \mathrm{a}, \mathrm{b}$ to eqs $22 \mathrm{a}, \mathrm{b}$, and obtain

$$
\begin{aligned}
& \Gamma_{k}^{\left(\alpha_{p}=0\right)} \approx w_{k} \Gamma_{p}^{\left(\alpha_{p}=0\right)} \\
& \Gamma_{k}^{\left(\alpha_{p}=1\right)} \approx w_{k} \Gamma_{p}^{\left(\alpha_{p}=1\right)}
\end{aligned}
$$

We can now insert eqs 28a,b into eq 24 and obtain

$$
\begin{array}{r}
f \approx\left[\Gamma_{\mathrm{p}}{ }^{\left(\alpha_{\mathrm{p}}=0\right)}+\left(\Gamma_{\mathrm{p}}{ }^{\left(\alpha_{\mathrm{p}}=1\right)}-\right.\right. \\
\left.\left.\Gamma_{\mathrm{p}}^{\left(\alpha_{\mathrm{p}}=0\right)}\right) \alpha_{\mathrm{p}}\right] \sum_{k=1}^{N} w_{k} \operatorname{erf}\left(y / \sqrt{\Lambda_{k}}\right)+\left[1-\Gamma_{p}^{\left(\alpha_{p}=0\right)}-\right. \\
\left.\quad\left(\Gamma_{p}^{\left(\alpha_{p}=1\right)}-\Gamma_{p}^{\left(\alpha_{p}=0\right)}\right) \alpha_{p}\right] \operatorname{erf}\left(y / \sqrt{\Lambda_{s}}\right)
\end{array}
$$

We are now in position to formally define a corrected refractiveindex function, $f^{*}\left(y, \alpha_{p}\right)$ by

$$
f^{*} \equiv f-\left[\Gamma_{p}^{\left(\alpha_{p}=0\right)}+\left(\Gamma_{p}^{\left(\alpha_{p}=1\right)}-\Gamma_{p}^{\left(\alpha_{p}=0\right)}\right) \alpha_{p}\right] \Omega
$$


where $\Omega \equiv \operatorname{erf}\left(y / \sqrt{\Lambda_{p}}\right)-\sum_{k=1}^{N} w_{k} \operatorname{erf}\left(y / \sqrt{\Lambda_{k}}\right)$, and $\Lambda_{p}$ is the smaller eigenvalue of the matrix of the four ternary diffusion coefficients. According to eq 29 and eq $30, f^{*}\left(y, \alpha_{p}\right)$ is approximately described by eq 20 . Hence, we can use $f^{*}\left(y, \alpha_{p}\right)$ to determine the four diffusion coefficients of the equivalent ternary system. If the deviation ${ }^{32} \Omega_{0}=\operatorname{erf}\left(y / \sqrt{D_{\mathrm{A}}}\right)-f$ is experimentally obtained for the macromolecule-solvent system, $\Omega(y)$ can be then evaluated using $\Omega\left(y / \Lambda_{p}^{1 / 2}\right)=\Omega_{0}\left(y / D_{\mathrm{A}}^{1 / 2}\right)$. However, $\Lambda_{p}, \Gamma_{p}{ }^{\left(\alpha_{p}=0\right)}$, and $\Gamma_{p}{ }^{\left(\alpha_{p}=1\right)}$ in eq 30 are not known initially. Hence, the four diffusion coefficients can be obtained from $f^{*}\left(y, \alpha_{p}\right)$ using eq 20 by successive approximations by initially setting $\Lambda_{p}=D_{\mathrm{A}}, \Gamma_{p}{ }^{\left(\alpha_{p}=1\right)}=1$ and $\Gamma_{p}{ }^{\left(\alpha_{p}=0\right)}=0$ in eq 30 .

Although we have neglected the cross-terms, $D_{i j}$, it is important to observe that this approximation is not necessary, and it has been introduced just to reduce mathematical complexity. The same results could have been obtained by replacing the $D_{i i}$ with the eigenvalues of the $N \times N$ diffusion-coefficient matrix, and the $C_{j}$ with the corresponding eigenvectors. Here, the only assumption is that these eigenvectors do not change in the presence of the additive.

\section{Materials and Methods}

Materials. Poly(ethylene glycol) samples with average molecular weights of 2, 8, and $20 \mathrm{~kg} \mathrm{~mol}^{-1}$ (PEG2k, PEG8k, and PEG20k, respectively) were purchased from Sigma-Aldrich and used without further purification. Poly(vinyl alcohol) (PVA, 99\% hydrolyzed) was purchased from Celanese Chemicals. The viscosity-average molecular weight of PVA, $M_{\mathrm{V}}$, was determined by viscosity measurements using the Mark-HouwinkSakurada equation: $[\eta]=K M_{\mathrm{V}}{ }^{a}$, where $K=40 \mathrm{~g} \mathrm{~L}^{-1}, a=$ 0.50 , and $[\eta]$ is the intrinsic viscosity. ${ }^{34}$ For our sample, $[\eta]=$ $0.0715 \mathrm{~L} \mathrm{~g}^{-1}$ and $M_{\mathrm{V}}=57 \mathrm{~kg} \mathrm{~mol}^{-1}$. Deionized water was passed through a four-stage Millipore filter system to provide high-purity water for all the experiments. All solutions were prepared by weight using a Mettler-Toledo AT400 analytical balance. Mass concentrations in grams per liter were obtained from the density of solutions. All density measurements $( \pm 1$ $\times 10^{-5} \mathrm{~g} \mathrm{~cm}^{-3}$ ) were made with a Mettler-Paar DMA40 density meter, thermostated with water from a large, well-regulated $\left( \pm 0.001{ }^{\circ} \mathrm{C}\right)$ water bath. The Rayleigh interferometry experiments require a pair of solutions with different composition. For each pair, we report their average mass concentration, $\bar{C}$ (in $\mathrm{g} \mathrm{L}^{-1}$ ) and the corresponding difference, $\Delta C$.

Rayleigh Interferometry. All diffusion measurements were made with the high-precision Gosting diffusiometer operated in its Rayleigh interferometric optical mode. A comprehensive description of the Gosting diffusiometer can be found in ref 19 and the references therein. In brief, a typical diffusion experiment using the Gosting diffusiometer starts with the preparation of a sharp boundary (using a peristaltic pump) between two uniform solutions of slightly different solute concentrations located inside a vertical channel with inside width $a$. In our case, we have $a=2.5057 \mathrm{~cm}$. The light source used for generating the Rayleigh interference pattern is a $\mathrm{He}-\mathrm{Ne}$ Uniphase laser with wavelength $\lambda=543.5 \mathrm{~nm}$. A cell holder is located inside a water bath. The temperature of the bath was regulated $\left( \pm 0.001{ }^{\circ} \mathrm{C}\right)$ at $25.00{ }^{\circ} \mathrm{C}$. The cell holder has the function to support a Tiselius cell, where diffusion occurs, and a mask, which consists of a double window. Here the laser beam is split into two parts: one going through the diffusion channel of the Tiselius cell and one passing through the water bath (reference channel). A pair of cylinder lenses focuses the diffusion channel onto the detector, where the Rayleigh interference pattern is observed and recorded. Rayleigh fringes shift
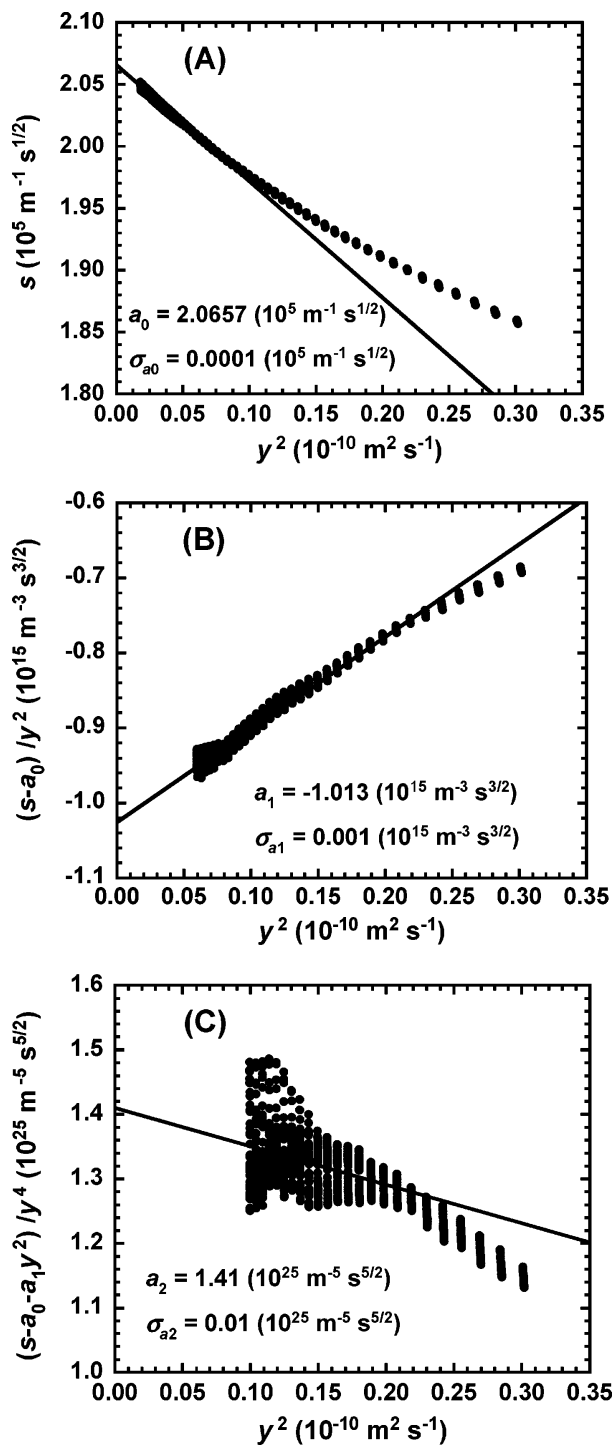

Figure 1. Determination of the parameters: $a_{0}, a_{1}$, and $a_{2}$ by linear extrapolation (solid lines) to $y^{2}=0$ of the functions: $s(\mathrm{~A}),\left(s-a_{0}\right) / y^{2}$ (B) and $\left(s-a_{0}-a_{1} y^{2}\right) / y^{4}(\mathrm{C})$, respectively. The illustrated case corresponds to the PVA-water system at $C=5.96 \mathrm{~g} \mathrm{~L}^{-1}$. The extrapolated values and the corresponding standard deviations are included.

horizontally as the refractive index inside the diffusion channel changes with vertical height. This gives direct information about refractive index versus vertical position. The difference in refractive index, $\Delta n$, between the two solutions is obtained from the total number of fringes $J$ using $\Delta n=J \lambda / a .^{30}$ The reported values of refractive-index increment, $R$, are calculated using $R$ $=\Delta n / \Delta C$.

The refractive-index profile inside the vertical channel, which is represented by $f(y) \equiv 2(n-\bar{n}) / \Delta n$, is extracted from the Rayleigh interference fringes at several times during boundary spreading. We have examined only the values of $f$ within the range $0.30 \leq f \leq 0.86$, taking into account the recommendation of Miller and Albright. ${ }^{30}$ We have then calculated $s=$ erfinv$(f) / y$. The values of the parameters $a_{0}, a_{1}$, and $a_{2}$ in eq 6 were obtained by linear extrapolation to $y^{2}=0$ of the functions $s$, $(s$ $\left.-a_{0}\right) / y^{2}$, and $\left(s-a_{0}-a_{1} y^{2}\right) / y^{4}$, respectively. Figure $1 \mathrm{a}-\mathrm{c}$ illustrate our approach. The range of $y^{2}$ was decreased until the values of $a_{0}, a_{1}$, and $a_{2}$, determined by linear extrapolation, were found to fluctuate within the experimental error. The relative standard deviations of $a_{0}, a_{1}$, and $a_{2}$ were found to be on the 
TABLE 1: Mean Diffusion Coefficients, $D_{\mathrm{A}}$, and Polydispersity Indices, $\omega$ and $\xi$, for Polymer-Water Systems

\begin{tabular}{lccccc}
\hline polymer & $\bar{C} /\left(\mathrm{g} \mathrm{L}^{-1}\right)$ & $\Delta C /\left(\mathrm{g} \mathrm{L}^{-1}\right)$ & $R /\left(\mathrm{g}^{-1} \mathrm{~mL}\right)$ & $D_{\mathrm{A}} /\left(10^{-5} \mathrm{~cm}^{2} \mathrm{~s}^{-1}\right)$ & $\omega$ \\
\hline PEG2k & 1.820 & 3.242 & 0.1320 & 0.1907 & 0.017 \\
PEG2k & 3.443 & 6.488 & 0.1319 & 0.1908 & 0.000 \\
PEG2k & 5.193 & 9.987 & 0.1321 & 0.1912 & 0.002 \\
PEG8k & 1.821 & 3.242 & 0.1339 & 0.08727 & 0.019 \\
PEG8k & 3.426 & 6.452 & 0.1350 & 0.09028 & 0.007 \\
PEG8k & 5.193 & 9.986 & 0.1338 & 0.05886 & 0.019 \\
PEG20k & 1.821 & 3.242 & 0.1343 & 0.05969 & 0.015 \\
PEG20k & 3.443 & 6.488 & 0.1342 & 0.06143 & 0.013 \\
PEG20k & 5.193 & 9.987 & 0.1342 & 0.06586 & 0.018 \\
PEG20k & 9.990 & 9.998 & 0.1345 & 0.07501 & 0.015 \\
PEG20k & 20.010 & 10.023 & 0.1342 & 0.02407 & 0.346 \\
PVA & 1.821 & 3.243 & 0.1437 & 0.02374 & 0.345 \\
PVA & 3.445 & 6.491 & 0.1439 & 0.02339 & 1.45 \\
PVA & 5.196 & 9.993 & 0.1440 & & 0.344
\end{tabular}

order of $0.01 \%, 0.1 \%$, and $1 \%$, respectively. We expect, however, that the actual errors are about 1 order of magnitude higher, due to other sources of errors such as boundary imperfections, aberration, the concentration dependence of diffusion coefficients, and solution preparation. The values of $D_{\mathrm{A}}, \omega$, and $\xi$ were then calculated using eqs $10-12$.

Dynamic Light Scattering. Measurements of dynamic light scattering were performed at $25.0 \pm 0.1{ }^{\circ} \mathrm{C}$. PEG-water samples were filtered through a $0.02-\mu \mathrm{m}$ filter (Anotop 10, Whatman). PVA-water samples were filtered through a $0.2 \mu \mathrm{m}$ filter (Anotop 10, Whatman) because of the presence of large clusters that clog the $0.02-\mu \mathrm{m}$ filters. The experiments were performed on a light scattering apparatus built using the following main components: a $\mathrm{He}-\mathrm{Ne}$ laser $(35 \mathrm{~mW}, 632.8$ $\mathrm{nm}$, Coherent Radiation), a manual goniometer and thermostat (Photocor Instruments), a multi-tau correlator, and an APD detector and software (PD4042, Precision Detectors).

In a typical DLS geometry, light coming from a laser is scattered by a sample and is collected at a given angle, $\theta$ (usually $90^{\circ}$ ) by a photodetector. The scattering angle defines the direction of the scattering vector: $q=\left(4 \pi n / \lambda_{0}\right) \sin (\theta / 2)$, where $\lambda_{0}$ is the wavelength of light in vacuum and $n$ is the refractive index of the sample. ${ }^{6}$ The scattered-intensity correlation functions were analyzed using a regularization algorithm (Precision Deconvolve 32, Precision Detectors). The application of regularization to the experimental correlation function is described in ref 38 and the references therein.

Computer Simulations. Computer simulations were performed using Matlab R2006a. We have simulated, to some extent, the actual experimental data-acquisition procedure employed on the Gosting diffusiometer. We have computed $f\left(y, \alpha_{p}\right)$ for a polydisperse macromolecule $(p)$-additive $(s)-$ solvent system with known $R_{p} / R_{s}$ and $(N+1) \times(N+1)$ diffusion-coefficient matrix. The four diffusion coefficients for the equivalent ternary system were defined for this system. For a given $\alpha_{p}$ value, we have generated 3000 equidistant $y / D^{\prime 1 / 2}$ values, where $1 / D^{\prime 1 / 2}=\alpha_{p} / D_{p p}{ }^{1 / 2}+\left(1-\alpha_{p}\right) / D_{s s}{ }^{1 / 2}$, and $0 \leq y /$ $D^{\prime 1 / 2} \leq 5$. We then computed $f\left(y, \alpha_{p}\right)+\delta f$, where $f\left(y, \alpha_{p}\right)$ is obtained using eq 24, and $\delta f$ is a random error sampled from a normal distribution with standard deviation $5 \times 10^{-5}$. This value falls within the range of statistical errors observed experimentally. Using eq 2 , we then computed $f(y, 1)+\delta f$ and, consequently, $\Omega_{0}=\operatorname{erf}\left(y / D_{\mathrm{A}}{ }^{1 / 2}\right)-(f+\delta f)$ for the corresponding macromolecule-solvent system with known diffusion-coefficient matrix. The source of error, $\delta f$, was included in order to differentiate between systematic errors associated with our approximate procedure and random errors associated with fitting sensitivity. To determine the four ternary diffusion coefficients, we applied the method of nonlinear least-squares to the uncorrected and the corrected values of $f\left(y, \alpha_{p}\right)+\delta f$ for two chosen $\alpha_{p}$ values and $0.30 \leq f \leq 0.86$ using eq 20 . The relative differences between the determined and the actual ternary diffusion coefficients were calculated for the uncorrected and corrected cases. By repeating each simulation 10 times, we have reported the mean values of the relative differences. To characterize statistical uncertainty, we have also included the corresponding confidence intervals using the student's $t$ test with $95 \%$ confidence level. These values describe the statistical errors associated with the obtained relative differences.

\section{Results and Discussion}

Effect of Macromolecule Polydispersity on Diffusion Coefficients of Macromolecule-Solvent Systems. Determination of $D_{A}, \omega$, and $\xi$ for Polymer-Solvent Systems. We have performed diffusion measurements on PEG-water (PEG2k, PEG8k, and PEG20k) and PVA-water systems at $25^{\circ} \mathrm{C}$ and several concentrations. In Table 1, we report the corresponding experimental values of $D_{\mathrm{A}}, \omega$, and $\xi$. In all cases, we have found that $\omega$ and $\xi$ are virtually independent of polymer concentration within the experimental error. As a diagnostic, we have also included the values of refractive-index increment, $R$. In the case of PEG, we have found that the differences between the $R$ values are all smaller than about $2 \%$. We can therefore conclude that $R$ is virtually independent of PEG molecular weight.

For all three PEG systems, the values of $\omega(\omega<0.02)$ are significantly smaller than those found for the PVA system $(\omega$ $\approx 0.35$ ). These results are expected since PEG approximately follows the narrow Poisson distribution, whereas PVA is expected to approximately follow the broader Flory distribution function. ${ }^{33,34}$ However, using eq 18 with $m=h=1$ and $a=$ 0.50 , we predict $\omega=0.106$ for PVA. This value is significantly lower than that obtained from our diffusion measurements. This apparent discrepancy can be understood by taking into consideration that PVA forms clusters in aqueous solutions. ${ }^{39,40}$ Clearly, these clusters have significantly lower diffusion coefficients. Thus, the presence of PVA free chains together with clusters can produce a significant increase in polydispersity. Our DLS measurements on PVA-water solutions, which will be discussed later, confirm the presence of clusters.

The accuracy of our $\omega$ and $\xi$ values can be examined by performing diffusion measurements on polydisperse PEG mixtures with known weight distribution, $w_{k}$. Since our PEG-water systems are nearly monodisperse, we have performed diffusion measurements on four PEG mixtures using the three different PEG molecular weights reported in Table 1. Our experimental values of $D_{\mathrm{A}}, \omega$, and $\xi$ are reported in Table 2 . We have also 


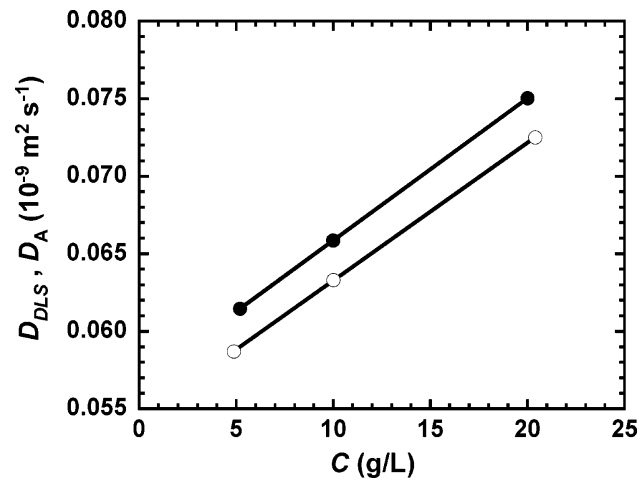

Figure 2. Mean diffusion coefficients: $D_{\mathrm{DLS}}(\bigcirc)$ and $D_{\mathrm{A}}(\bullet)$ for the PEG20k-water system as a function of polymer concentration, $C$. Solid lines are linear fits through the data.

TABLE 2: Experimental (exp) and Calculated (calc) Values of $D_{\mathrm{A}}, \omega$, and $\xi$ for PEG-Water Polydisperse Systems

\begin{tabular}{lllll}
\hline $\bar{C} /\left(\mathrm{g} \mathrm{L}^{-1}\right)$ & 3.643 & 3.643 & 3.643 & 5.467 \\
$w$ (PEG2k) & 0.5000 & 0.0000 & 0.5000 & 0.3333 \\
$w($ PEG8k) & 0.5000 & 0.5000 & 0.0000 & 0.3333 \\
$w($ PEG20k) & 0.0000 & 0.5000 & 0.5000 & 0.3333 \\
$\Delta C /\left(\mathrm{g} \mathrm{L}^{-1}\right)$ & 6.488 & 6.488 & 6.489 & 9.739 \\
$R /\left(\mathrm{g}^{-1} \mathrm{~mL}\right)$ & 0.1332 & 0.1343 & 0.1334 & 0.1336 \\
$D_{\mathrm{A}} /\left(10^{-5} \mathrm{~cm}^{2} \mathrm{~s}^{-1}\right)(\exp )$ & 0.1265 & 0.07387 & 0.09960 & 0.09572 \\
$\omega(\exp )$ & 0.117 & 0.048 & 0.275 & 0.172 \\
$\xi(\exp )$ & 0.41 & 0.16 & 1.07 & 0.58 \\
$D_{\mathrm{A}} /\left(10^{-5} \mathrm{~cm}^{2} \mathrm{~s}^{-1}\right)($ calc) & 0.1242 & 0.07098 & 0.09730 & 0.09377 \\
$\omega$ (calc) & 0.112 & 0.029 & 0.245 & 0.156 \\
$\xi$ (calc) & 0.38 & 0.10 & 0.85 & 0.52
\end{tabular}

calculated these three parameters by applying eqs $10-12$ to the experimental $w_{k}$ in Table 2 and the corresponding PEG diffusion coefficients of Table 1 at $w_{k} \bar{C}$. These values, which are also reported in Table 2, were found to be systematically smaller than the experimental ones. This difference ranges from 0.005 to 0.03 for $\omega$, and from 0.03 to 0.22 for $\xi$. Clearly, only part of this discrepancy can be attributed to the small polydispersity of individual PEG samples. We therefore estimate that the accuracies of $\omega$ and $\xi$ are $\sim 0.01$ and $\sim 0.1$, respectively.

Comparison between Rayleigh Interferometry and Dynamic Light Scattering. We have also performed DLS measurements on PEG20k-water and PVA-water systems at $25^{\circ} \mathrm{C}$. Our DLS results will be directly compared with those obtained by Rayleigh interferometry. It is, however, worthwhile to first briefly review some theoretical aspect of DLS relevant to the comparison between these two different techniques and to polydispersity.

The DLS technique yields the electric-field correlation function $g^{(1)}(\tau)=\left\langle E(t) E^{*}(t+\tau)\right\rangle /\left\langle E(t) E^{*}(t)\right\rangle$ associated with stochastic temporal fluctuations of the electric field, $E(t)$, scattered at a given angle defined by the scattering vector $q$ (see Materials and Methods). ${ }^{6}$ In the commonly employed homodyne mode, this is achieved by monitoring the temporal fluctuations of light intensity $I(t)$ at the scattering angle. For a polydisperse particles in dilute solution, we can write ${ }^{7}$

$$
g^{(1)}(\tau)=\sum_{k=1}^{N}\left(i_{S}\right)_{k} \mathrm{e}^{-\gamma_{k}(q) \tau}
$$

where $\left(i_{S}\right)_{k}=P_{k}(q) w_{k} M_{k}$ is the light-scattering contribution of the macromolecular component $k$, and $P_{k}(q) \leq 1$ is its form factor. The $\gamma_{k}(q)$ values are relaxation rates that generally take into account not only particle center-of-mass diffusion but also
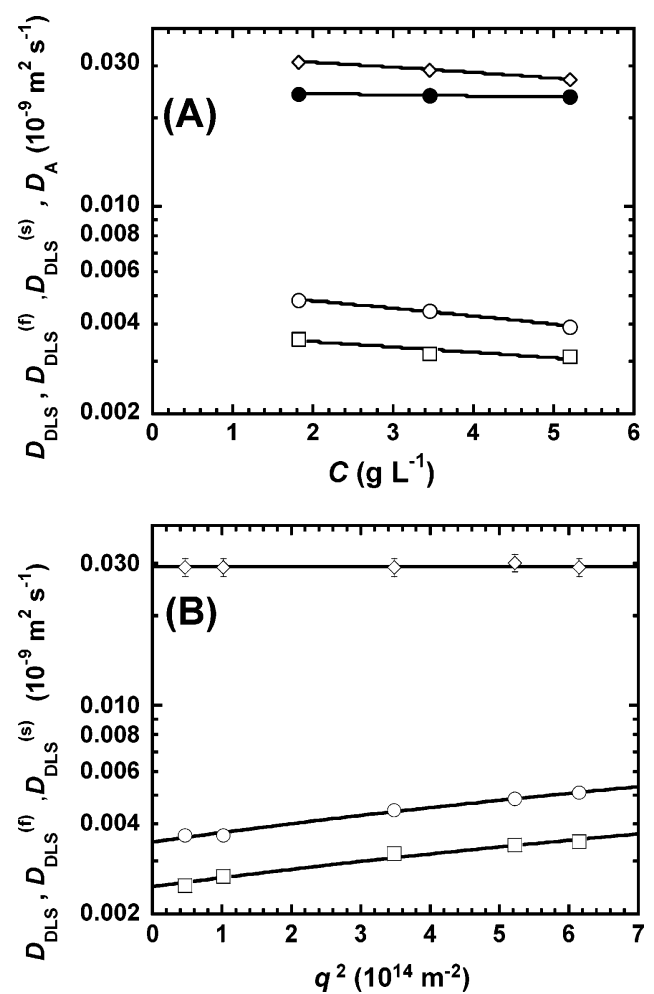

Figure 3. Mean diffusion coefficients, $D_{\text {DLS }}(\bigcirc), D_{\mathrm{DLS}}^{(\mathrm{f})}(\diamond), D_{\mathrm{DLS}}^{(\mathrm{s})}(\square)$ and $D_{\mathrm{A}}(\bullet)$ for the PVA-water system. (A) Mean diffusion coefficients as a function of polymer concentration, $C$ at the $90^{\circ}$ scattering angle. (B) Mean diffusion coefficients as a function of $q^{2}$ at $C=3.45 \mathrm{~g} \mathrm{~L}^{-1}$. Solid lines are linear fits through the data.

other, $q$-dependent, dynamic features (e.g., rotational diffusion of anisotropic particles) of individual particles. If the characteristic size of the particles is small compared to $q^{-1}$, then $P_{k^{-}}$ $(q)=P_{k}(0)=1$ and $\gamma_{k}(q)=\gamma_{k}(0)=q^{2} D_{k}$. Moreover, $\gamma_{k}(q)$ can be assumed to be equal to $q^{2} D_{k}$, even for large particles, provided that they do not display significant anisotropy. We shall adopt this assumption. The cumulant analysis introduced by $\mathrm{Koppel}^{41}$ describes polydisperse systems in terms of DLSbased diffusion moments. This analysis, which is analogous to that outlined above for Rayleigh interferometry, is based on the following power series expansion:

$$
\ln g^{(1)}(\tau)=-q^{2} \tau \sum_{i=0}^{\infty} \frac{(-1)^{i}}{(i+1) !} b_{i}\left(q^{2} \tau\right)^{i}
$$

where

$$
\begin{gathered}
\langle D\rangle_{z}=b_{0} \\
\left\langle D^{2}\right\rangle_{z}=b_{0}{ }^{2}+b_{1} \\
\left\langle D^{3}\right\rangle_{z}=b_{0}{ }^{3}+3 b_{0} b_{1}+b_{2}
\end{gathered}
$$

and

$$
\left\langle D^{n}\right\rangle_{z} \equiv \frac{\sum_{k=1}^{N} P_{k}(q) w_{k} M_{k} D_{k}{ }^{n}}{\sum_{k=1}^{N} P_{k}(q) w_{k} M_{k}}
$$


TABLE 3: Relative Percentage Differences for $D_{p p}, D_{p s}, D_{s p}$, and $D_{s s}$, with $D_{p s} / D_{s s}=D_{s p} / D_{s s}=1 / 100$

\begin{tabular}{|c|c|c|c|c|c|c|}
\hline \multirow[b]{2}{*}{$\omega$} & \multicolumn{2}{|c|}{$D_{p p} / D_{s s}=1 / 10$} & \multicolumn{2}{|c|}{$D_{p p} / D_{s s}=1 / 5$} & \multicolumn{2}{|c|}{$D_{p p} / D_{s s}=1 / 2$} \\
\hline & uncorrected & corrected & uncorrected & corrected & uncorrected & corrected \\
\hline 0000 & $000+001^{a}$ & $000+001$ & $\begin{array}{c}D_{p p} \\
\end{array}$ & $000+001$ & $001+002$ & $000+002$ \\
\hline 0.025 & $1.80 \pm 0.01$ & $0.01 \pm 0.01$ & $2.34 \pm 0.01$ & $0.01 \pm 0.01$ & $4.81 \pm 0.02$ & $0.06 \pm 0.03$ \\
\hline 0.050 & $3.50 \pm 0.01$ & $0.01 \pm 0.01$ & $4.58 \pm 0.01$ & $0.03 \pm 0.01$ & $9.32 \pm 0.02$ & $0.15 \pm 0.03$ \\
\hline 0.100 & $6.67 \pm 0.01$ & $0.02 \pm 0.01$ & $8.73 \pm 0.01$ & $0.05 \pm 0.01$ & $17.51 \pm 0.02$ & $0.37 \pm 0.03$ \\
\hline 0.200 & $12.24 \pm 0.01$ & $0.06 \pm 0.01$ & $16.03 \pm 0.01$ & $0.11 \pm 0.01$ & $31.50 \pm 0.02$ & $1.18 \pm 0.05$ \\
\hline $\begin{array}{l}0.000 \\
0.025 \\
0.050 \\
0.100 \\
0.200\end{array}$ & $\begin{array}{r}-0.1 \pm 0.2 \\
0.1 \pm 0.2 \\
0.1 \pm 0.2 \\
0.5 \pm 0.2 \\
1.0 \pm 0.2\end{array}$ & $\begin{array}{l}-0.1 \pm 0.2 \\
-0.2 \pm 0.2 \\
-0.4 \pm 0.2 \\
-0.5 \pm 0.2 \\
-0.6 \pm 0.2\end{array}$ & $\begin{array}{c}D_{p s} \\
0.1 \pm 0.2 \\
0.6 \pm 0.2 \\
1.0 \pm 0.2 \\
1.5 \pm 0.2 \\
2.4 \pm 0.2\end{array}$ & $\begin{array}{l}0.0 \pm 0.2 \\
0.0 \pm 0.2 \\
0.1 \pm 0.2 \\
0.3 \pm 0.2 \\
1.9 \pm 0.2\end{array}$ & $\begin{array}{r}0 \pm 1 \\
1 \pm 1 \\
0 \pm 1 \\
-1 \pm 1 \\
-1 \pm 1\end{array}$ & $\begin{aligned} 0 & \pm 1 \\
5 & \pm 2 \\
7 & \pm 1 \\
13 & \pm 1 \\
23 & \pm 1\end{aligned}$ \\
\hline $\begin{array}{l}0.000 \\
0.025 \\
0.050 \\
0.100 \\
0.200\end{array}$ & $\begin{array}{r}-0.1 \pm 0.3 \\
-134.1 \pm 0.4 \\
-262.5 \pm 0.5 \\
-504.9 \pm 0.6 \\
-940.8 \pm 0.3\end{array}$ & $\begin{array}{r}0.3 \pm 0.7 \\
-0.4 \pm 0.4 \\
-0.8 \pm 0.7 \\
-1.5 \pm 0.6 \\
-4.0 \pm 0.4\end{array}$ & $\begin{aligned} & D_{s p} \\
-0.3 & \pm 0.5 \\
-182.3 & \pm 0.6 \\
-357.3 & \pm 0.4 \\
-685.6 & \pm 0.7 \\
-1271.3 & \pm 0.4\end{aligned}$ & $\begin{array}{l}-0.2 \pm 0.6 \\
-0.6 \pm 0.9 \\
-2.2 \pm 0.6 \\
-3.8 \pm 0.8 \\
-8.2 \pm 0.7\end{array}$ & $\begin{array}{r}-1 \pm 2 \\
-419 \pm 1 \\
-813 \pm 1 \\
-1532 \pm 1 \\
-2764 \pm 1\end{array}$ & $\begin{array}{r}-1 \pm 2 \\
-5 \pm 2 \\
-13 \pm 2 \\
-32 \pm 2 \\
-100 \pm 4\end{array}$ \\
\hline $\begin{array}{l}0.000 \\
0.025 \\
0.050 \\
0.100 \\
0.200\end{array}$ & $\begin{array}{r}0.000 \pm 0.004 \\
-0.007 \pm 0.003 \\
-0.014 \pm 0.002 \\
-0.030 \pm 0.003 \\
-0.040 \pm 0.003\end{array}$ & $\begin{array}{r}0.003 \pm 0.004 \\
-0.001 \pm 0.003 \\
-0.003 \pm 0.003 \\
-0.012 \pm 0.003 \\
-0.037 \pm 0.003\end{array}$ & $\begin{array}{c}D_{s s} \\
-0.002 \pm 0.004 \\
-0.013 \pm 0.004 \\
-0.023 \pm 0.003 \\
-0.036 \pm 0.005 \\
-0.052 \pm 0.005\end{array}$ & $\begin{array}{l}-0.001 \pm 0.003 \\
-0.010 \pm 0.004 \\
-0.020 \pm 0.004 \\
-0.041 \pm 0.004 \\
-0.106 \pm 0.004\end{array}$ & $\begin{aligned} 0.00 & \pm 0.02 \\
-0.01 & \pm 0.02 \\
0.00 & \pm 0.01 \\
-0.01 & \pm 0.01 \\
-0.01 & \pm 0.01\end{aligned}$ & $\begin{array}{r}0.00 \pm 0.01 \\
-0.08 \pm 0.03 \\
-0.13 \pm 0.01 \\
-0.23 \pm 0.01 \\
-0.40 \pm 0.01\end{array}$ \\
\hline
\end{tabular}

${ }^{a}$ Confidence intervals using the student's $t$ test with 95\% confidence level as described in Materials and Methods.

TABLE 4: Relative Percentage Differences on $D_{s p}$ for $D_{p p} / D_{s s}=1 / 10$ and $D_{p s} / D_{s s}=1 / 100$

\begin{tabular}{crrrr}
\hline & $D_{s p} / D_{s s}=$ & \multicolumn{1}{c}{$D_{s p} / D_{s s}=$} & \multicolumn{1}{c}{$D_{s p} / D_{s s}=$} & \multicolumn{1}{c}{$D_{s p} / D_{s s}=$} \\
$\omega$ & $1 / 1000$ & $1 / 100$ & \multicolumn{1}{c}{$1 / 10$} & \multicolumn{1}{c}{$-1 / 100$} \\
\hline 0.000 & $3 \pm 4$ & $0.3 \pm 0.7$ & $0.01 \pm 0.03$ & $0.0 \pm 0.7$ \\
0.025 & $0 \pm 6$ & $-0.4 \pm 0.4$ & $-0.41 \pm 0.05$ & $-0.5 \pm 0.7$ \\
0.050 & $0 \pm 7$ & $-0.8 \pm 0.7$ & $-0.87 \pm 0.04$ & $-0.6 \pm 0.6$ \\
0.100 & $-5 \pm 7$ & $-1.5 \pm 0.6$ & $-1.81 \pm 0.03$ & $-1.4 \pm 0.6$ \\
0.200 & $-3 \pm 6$ & $-4.0 \pm 0.4$ & $-3.77 \pm 0.04$ & $-3.4 \pm 0.4$
\end{tabular}

The DLS mean diffusion coefficient, $D_{\mathrm{DLS}}$, is the $z$-average diffusion coefficient: ${ }^{7}$

$$
D_{\mathrm{DLS}} \equiv\langle D\rangle_{z}=b_{0}
$$

Polydispersity can be quantified using the diffusion momenta of higher order. For example, the relative variance

$$
\sigma^{2} \equiv \frac{\left\langle D^{2}\right\rangle_{z}}{\langle D\rangle_{z}^{2}}-1=\frac{b_{1}}{b_{0}^{2}}
$$

is used as the DLS polydispersity index. One important feature of DLS compared to classical techniques is that, according to eq 34, high-molecular weight components contribute more significantly to $D_{\mathrm{DLS}}$ than to $D_{\mathrm{A}}$. Hence it is expected that $D_{\mathrm{A}} /$ $D_{\text {DLS }}>1$ for polydisperse systems.

In Figure 2, we show the values of $D_{\mathrm{A}}$ and $D_{\text {DLS }}$ obtained for PEG20k-water binary solutions. The DLS data were obtained at the $90^{\circ}$ scattering angle. We find that $D_{\mathrm{A}} / D_{\mathrm{DLS}}=$ 1.04 within our experimental concentration range. To estimate the contribution of PEG polydispersity to $D_{\mathrm{A}} / D_{\mathrm{DLS}}$, we have first determined the scaling factor, $a$, by reporting our $D_{\mathrm{A}}$ values as a function of PEG molecular weight. We have obtained $a=$ 0.53 in agreement with literature. We have then constructed the $w_{k}$ distribution function using eq 13 with $m=1$ and $h=$ 12. This distribution corresponds to $\omega=0.016$ consistent with the $\omega$ values reported in Table 1 for PEG20k. We have not
TABLE 5: Relative Percentage Differences on $D_{s p}$ for $D_{p p} / D_{s s}=1 / 10$ and $D_{p s} / D_{s s}=D_{s p} / D_{s s}=1 / 100$

\begin{tabular}{cccc}
\hline$\omega$ & $\alpha_{p}=0.2,0.8$ & $\alpha_{p}=0.5,1$ & $\alpha_{p}=0,0.5$ \\
\hline \multicolumn{4}{c}{$D_{p p} / D_{s s}=1 / 10$} \\
0.000 & $0.1 \pm 0.3$ & $-0.1 \pm 0.2$ & $0.1 \pm 0.5$ \\
0.025 & $-0.6 \pm 0.3$ & $-0.5 \pm 0.3$ & $0.0 \pm 0.4$ \\
0.050 & $-0.9 \pm 0.3$ & $-0.9 \pm 0.2$ & $-0.9 \pm 0.7$ \\
0.100 & $-2.0 \pm 0.4$ & $-1.9 \pm 0.2$ & $-1.9 \pm 0.4$ \\
0.200 & $-4.1 \pm 0.4$ & $-4.6 \pm 0.2$ & $-3.9 \pm 0.3$ \\
0 & \multicolumn{2}{c}{$D_{p p} / D_{s s}=1 / 2$} \\
0.000 & $0 \pm 2$ & $-1 \pm 3$ & $0 \pm 2$ \\
0.025 & $-6 \pm 2$ & $-6 \pm 3$ & $-6 \pm 2$ \\
0.050 & $-14 \pm 2$ & $-14 \pm 2$ & $-14 \pm 2$ \\
0.100 & $-35 \pm 3$ & $-34 \pm 4$ & $-32 \pm 2$ \\
0.200 & $-106 \pm 3$ & $-124 \pm 5$ & $-99 \pm 2$
\end{tabular}

used the Poisson distribution because its corresponding $\omega$ value $\left(\omega=5 \times 10^{-4}\right)$ was very low compared to our experimental results. Using eq 10 and eq 35 , we have then calculated $D_{\mathrm{A}} /$ $D_{\text {DLS }}=1.025$. Here we have assumed $P_{k}(q)=1$ since the size of PEG20k molecules is small compared to $q^{-1}$. This estimation on polydispersity allows us to conclude that the $D_{\text {DLS }}$ values agree with the $D_{\mathrm{A}}$ values obtained by Rayleigh interferometry within an acceptable error of about $2 \%$.

Interestingly, a large discrepancy between $D_{\mathrm{A}}$ and $D_{\mathrm{DLS}}$ experimental values were observed for the PVA-water system. For the DLS technique, the well-established regularization algorithm ${ }^{38}$ was applied to eq 31 for the determination of the $\left(i_{S}\right)_{k}$ distribution of diffusion coefficients, $D_{k}$. We have found that $\left(i_{S}\right)_{k}$ consists of two well-separated peaks and calculated the corresponding two mean diffusion coefficients, $D_{\mathrm{DLS}}^{(\mathrm{f})}$ and $D_{\mathrm{DLS}}^{(\mathrm{s})}$, using eq 35 . The values of $D_{\mathrm{DLS}}^{(\mathrm{f})}$ are consistent with the size of PVA molecules $(\sim 10 \mathrm{~nm})$. However, the values of $D_{\mathrm{DLS}}^{(\mathrm{s})}$ are significantly lower and correspond to the PVA clusters $(\sim 100 \mathrm{~nm})$ mentioned above. In Figure 3a, we show the $D_{\text {DLS }}, D_{\text {DLS }}^{(\mathrm{f})}, D_{\text {DLS }}^{(\mathrm{s})}$ values obtained at the $90^{\circ}$ scattering angle together with the corresponding $D_{\mathrm{A}}$ values reported in Table 1 . We find that $D_{\mathrm{A}} / D_{\text {DLS }} \approx 5$ within our experimental 
TABLE 6: Relative Percentage Differences on $D_{s p}$ for $D_{s p} / D_{s s}=1 / 100$

\begin{tabular}{|c|c|c|c|c|c|c|}
\hline$\omega$ & $D_{p p} / D_{s s}=1 / 100$ & $D_{p p} / D_{s s}=1 / 10$ & $D_{p p} / D_{s s}=1 / 5$ & $D_{p p} / D_{s s}=1 / 4$ & $D_{p p} / D_{s s}=1 / 3$ & $D_{p p} / D_{s s}=1 / 2$ \\
\hline $\begin{array}{l}0.000 \\
0.025 \\
0.050 \\
0.100 \\
0.200\end{array}$ & $\begin{array}{l}0.0 \pm 0.3 \\
0.0 \pm 0.3 \\
0.2 \pm 0.5 \\
0.1 \pm 0.3 \\
0.7 \pm 0.3\end{array}$ & $\begin{array}{l}-0.6 \pm 0.7 \\
-0.1 \pm 0.4 \\
-0.3 \pm 0.9 \\
-0.3 \pm 0.5 \\
-1.2 \pm 0.4\end{array}$ & $\begin{array}{l}D_{p s} / D_{p p}=-1 / 5 \\
-0.4 \pm 0.8 \\
-0.6 \pm 0.8 \\
-1.4 \pm 0.8 \\
-2.2 \pm 0.8 \\
-5.6 \pm 0.9\end{array}$ & $\begin{array}{r}0 \pm 1 \\
0 \pm 1 \\
-2 \pm 1 \\
-3 \pm 1 \\
-8 \pm 1\end{array}$ & $\begin{array}{r}1 \pm 1 \\
-2 \pm 1 \\
-3 \pm 1 \\
-7 \pm 1 \\
-18 \pm 1\end{array}$ & $\begin{array}{r}0 \pm 2 \\
-5 \pm 2 \\
-12 \pm 3 \\
-32 \pm 3 \\
-132 \pm 5\end{array}$ \\
\hline $\begin{array}{l}0.000 \\
0.025 \\
0.050 \\
0.100 \\
0.200\end{array}$ & $\begin{array}{r}0.0 \pm 0.3 \\
0.0 \pm 0.3 \\
0.1 \pm 0.3 \\
-0.1 \pm 0.4 \\
-0.4 \pm 0.2\end{array}$ & $\begin{array}{r}0.1 \pm 0.5 \\
-0.2 \pm 0.6 \\
-1.3 \pm 0.5 \\
-1.9 \pm 0.6 \\
-2.7 \pm 0.3\end{array}$ & $\begin{array}{c}D_{p s} / D_{p p}=0 \\
0.5 \pm 0.8 \\
-0.6 \pm 0.7 \\
-1.8 \pm 0.9 \\
-3.6 \pm 0.6 \\
-7.5 \pm 0.7\end{array}$ & $\begin{array}{r}0 \pm 1 \\
-1 \pm 2 \\
-3 \pm 1 \\
-6 \pm 1 \\
-11 \pm 1\end{array}$ & $\begin{array}{r}0 \pm 1 \\
-2 \pm 2 \\
-5 \pm 1 \\
-9 \pm 1 \\
-22 \pm 1\end{array}$ & $\begin{array}{r}0 \pm 2 \\
-6 \pm 3 \\
-14 \pm 2 \\
-31 \pm 3 \\
-103 \pm 3\end{array}$ \\
\hline $\begin{array}{l}0.000 \\
0.025 \\
0.050 \\
0.100 \\
0.200\end{array}$ & $\begin{array}{r}0.1 \pm 0.4 \\
-0.2 \pm 0.2 \\
-0.2 \pm 0.4 \\
-0.7 \pm 0.4 \\
-1.5 \pm 0.3\end{array}$ & $\begin{array}{r}0.1 \pm 0.6 \\
-0.8 \pm 0.5 \\
-1.2 \pm 0.6 \\
-2.2 \pm 0.7 \\
-4.3 \pm 0.3\end{array}$ & $\begin{array}{c}D_{p s} / D_{p p}=1 / 5 \\
0.2 \pm 0.9 \\
-1.2 \pm 0.7 \\
-1.9 \pm 0.8 \\
-5.0 \pm 0.7 \\
-9.7 \pm 0.9\end{array}$ & $\begin{array}{r}0 \pm 1 \\
-1 \pm 1 \\
-3 \pm 1 \\
-6 \pm 1 \\
-13 \pm 1\end{array}$ & $\begin{array}{r}0 \pm 1 \\
-2 \pm 1 \\
-4 \pm 1 \\
-11 \pm 1 \\
-25 \pm 1\end{array}$ & $\begin{array}{r}1 \pm 2 \\
-7 \pm 1 \\
-14 \pm 1 \\
-31 \pm 2 \\
-90 \pm 3\end{array}$ \\
\hline
\end{tabular}

concentration range. We also observe that $D_{\mathrm{DLS}}$ is closer to $D_{\mathrm{DLS}}^{(\mathrm{s})}$, whereas $D_{\mathrm{A}}$ is closer to $D_{\mathrm{DLS}}^{(\mathrm{f})}$. Since $D_{\mathrm{DLS}}^{(\mathrm{s})}$ corresponds to very large clusters, the $P_{k}(q)$ values at $90^{\circ}$ are expected to be appreciably smaller than $P_{k}(0)=1$. We have therefore performed DLS measurements at several angles. Our results are shown in Figure $3 \mathrm{~b}$ for PVA at the representative concentration: $C=3.45 \mathrm{~g} \mathrm{~L}^{-1}$. As expected, we can see that $D_{\mathrm{DLS}}^{(\mathrm{f})}$ is independent of $q^{2}$ within the experimental error. On the other hand $D_{\mathrm{DLS}}^{(\mathrm{s})}$ increase with $q^{2}$. This occurs because a large cluster $l$ contribute less to $D_{\mathrm{DLS}}^{(\mathrm{s})}$ compared to small clusters $s$, due the corresponding more significant decrease of $P_{l}(q)$ compared to $P_{s}(q)$ as $q^{2}$ increases. Hence, in the limit of $q$ approaching zero, discrepancy between $D_{\mathrm{A}}$ and $D_{\mathrm{DLS}}$ was found to be even larger $\left(D_{\mathrm{A}} / D_{\mathrm{DLS}} \approx 10\right)$. In conclusion this comparison clearly illustrates how DLS and Rayleigh interferometry provide two significantly different results for macromolecules with large polydispersity.

Effect of Macromolecular Polydispersity on Ternary Diffusion Coefficients of Macromolecule-Additive-Solvent Systems. Simulation on a Model Polydisperse System. We have described an approximate procedure to obtain the four ternary diffusion coefficients from macromolecule $(p)-\operatorname{additive}(s)-$ solvent systems in the presence of polydispersity. Here we examine its accuracy using computer simulations (see Materials and Methods) on a model polydisperse system. We consider a simple macromolecular solute consisting of three macromolecular components with weight fractions $w_{1}=1 / 6, w_{2}=2 / 3$, and $w_{3}=1 / 6$. The corresponding three main-diffusion coefficients are $D_{p p} /\left(1+\omega^{1 / 2}\right)^{2}, D_{p p}$, and $D_{p p} /\left(1-\omega^{1 / 2}\right)^{2}$, where $D_{p p}$ is their mean diffusion coefficient and $\omega$ the polydispersity index previously defined by eq 11 . The diffusion-coefficient matrix for this model system is

$$
\begin{aligned}
& {\left[\begin{array}{llll}
D_{11} & D_{12} & D_{13} & D_{1 \mathrm{~s}} \\
D_{21} & D_{22} & D_{23} & D_{2 \mathrm{~s}} \\
D_{31} & D_{32} & D_{33} & D_{3 \mathrm{~s}} \\
D_{\mathrm{s} 1} & D_{\mathrm{s} 2} & D_{\mathrm{s} 3} & D_{\mathrm{ss}}
\end{array}\right]=} \\
& \\
& {\left[\begin{array}{llll}
D_{\mathrm{pp}} /\left(1+\omega^{1 / 2}\right)^{2} & 0 & 0 & \\
0 & D_{p p} & 0 & \\
0 & 0 & D_{\mathrm{pp}} /\left(1-\omega^{1 / 2}\right)^{2} & w_{3} D_{\mathrm{ps}} \\
D_{s p} & D_{s p} & D_{s p} & D_{s s}
\end{array}\right]}
\end{aligned}
$$

where $D_{p p}, D_{p s}, D_{s p}$, and $D_{s s}$ are the diffusion coefficients of the equivalent ternary system. For the corresponding macromolecule-solvent system, we have assumed the following diffusion-coefficient matrix:

$$
\begin{aligned}
{\left[\begin{array}{lll}
D_{11} & D_{12} & D_{13} \\
D_{21} & D_{22} & D_{23} \\
D_{31} & D_{32} & D_{33}
\end{array}\right] } & = \\
\eta & {\left[\begin{array}{lll}
D_{\mathrm{pp}} /\left(1+\omega^{0.5}\right)^{2} & 0 & 0 \\
0 & D_{p p} & 0 \\
0 & 0 & D_{\mathrm{pp}} /\left(1-\omega^{0.5}\right)^{2}
\end{array}\right] }
\end{aligned}
$$

where $\eta$ is a coefficient that takes into account the effect of the additive on the macromolecule diffusion coefficients. We have found that $\eta$ has a negligible effect on our results. Hence, we will assume $\eta=1$ in the following discussion.

We have calculated the relative deviations of the determined diffusion coefficients with respect to their actual values as a function $\omega$ with $0 \leq \omega \leq 0.2$. We note that $\omega=0.2$ corresponds to $M_{\mathrm{w}} / M_{\mathrm{n}}=3.5$ for our model system and $a=0.5$. This analysis was performed for several values of $D_{p p} / D_{s s}, D_{s p} / D_{s s}$ and $D_{p s} /$ $D_{s s}$ using $f\left(y, \alpha_{p}\right)$ with $\alpha_{p}=0$ and $\alpha_{p}=1$. In Table 3, we examine the effect of polydispersity on the accuracy of all four diffusion coefficients as a function of $D_{p p} / D_{s s}$ for the representative case $D_{s p} / D_{s s}=D_{p s} / D_{s s}=1 / 100$. The relative percent deviations (and corresponding statistical errors) were obtained for both uncorrected and corrected refractive-index profiles. We observe that reported deviations increase as $D_{p p} /$ $D_{s s}$ approaches to one. This is expected since our corrective procedure becomes more accurate as $D_{p p}$ becomes small compared to $D_{s s}$.

We can see that polydispersity has a negligible effect on $D_{s s}$ accuracy for the uncorrected and corrected cases. For $D_{p s}$, we find that polydispersity also produces a marginal discrepancies $(\leq 2 \%)$. However, we observe that our corrective procedure improves the accuracy of this coefficient for the cases with $D_{p p} /$ $D_{s s} \leq 1 / 5$ and fails to work for the case with $D_{p p} / D_{s s}=1 / 2$. In general, we have found that polydispersity has a marginal effect on the accuracy of the determined $D_{s s}$ and $D_{p s}$ values. On the other hand, the accuracy of the determined $D_{p p}$ and $D_{s p}$ values is significantly improved when using our corrective procedure. 
For $D_{p p}$, our correction virtually removes the effect of polydispersity completely. For $D_{p p} / D_{s s}=1 / 2$ and $\omega \geq 0.10$, the discrepancies are larger than $10 \%$ without correction and reduce to $1 \%$ or less after the correction is applied. For $D_{s p}$, correction becomes essential even for the lowest polydispersity case: $\omega$ $=0.025$ and $D_{p p} / D_{s s}=1 / 10$. Here the correction reduces the error from more than $100 \%$ to less than $1 \%$. Moreover, our corrective procedure generally produces discrepancies lower than $10 \%$ with the exception of the $D_{p p} / D_{s s}=1 / 2$ cases with $\omega$ $\geq 0.05$. In the following analysis, we will focus on this diffusion coefficient, since the accuracy of $D_{s p}$ is significantly affected by polydispersity.

We have examined the role of $D_{s p} / D_{s s}$ magnitude and sign on the accuracy of $D_{s p}$. In Table 4 , we can see that the accuracy of our corrective procedure is not affected by the magnitude and sign of $D_{s p} / D_{s s}$. We observe, however, that the statistical error of the reported deviations increases as the magnitude of $D_{s p} / D_{s s}$ decreases. This is expected since the determination of $D_{s p}$ becomes more difficult as the effect of this coefficient on the refractive-index profile becomes comparable with statistical noise. In Table 5, we have examined the effect of different choices of $\alpha_{p}$ pairs on the accuracy of our results for the representative two cases: $D_{p p} / D_{s s}=1 / 10,1 / 2$ with $D_{s p} / D_{s s}=D_{p s} / D_{s s}=1 / 100$. These results can be directly compared with those in Table 3. We find that the choice of $\alpha_{p}$ pair has a marginal effect on the accuracy of the corrective procedure.

Finally, Table 6 provides a broad spectrum of relative difference for $D_{s p}$. We report several cases within the ranges $1 / 1000 \leq D_{p p} / D_{s s} \leq 1 / 2$ and $-1 / 5 \leq D_{p s} / D_{p p} \leq 1 / 5$. We notice that the accuracy of our corrective procedure improves as $D_{p s} /$ $D_{p p}$ from positive becomes negative. In all examined cases, $D_{p p} /$ $D_{s s}$ remains the most important factor affecting the accuracy of our corrective procedure.

\section{Summary and Conclusions}

We have reported novel equations for the extraction of diffusion moments from the Rayleigh interferometric pattern. We have experimentally determined mean diffusion coefficients and two polydispersity parameters for aqueous solutions of PEG and PVA at $25^{\circ} \mathrm{C}$. Aqueous solutions of PEG mixtures were used to examine the accuracy of the polydispersity parameters. We have found that the accuracies of $\omega$ and $\xi$ are $\sim 0.01$ and $\sim 0.1$, respectively. We have compared diffusion coefficients obtained using Rayleigh interferometry and dynamic light scattering. We have found that these two techniques are in good agreement with each other in the case of PEG20k, which is a polymer with a very low polydispersity. On the other hand, we have found a significant discrepancy between these two techniques in the case of PVA due to the presence of polymer clusters, which are responsible for a large diffusion polydispersity. Finally, for two-solute mixtures with one polydisperse solute, we have reported a novel corrective procedure for extracting accurate ternary diffusion coefficients from Rayleigh interferometry. Our computer simulations show that polydispersity has a small effect on the accuracy of $D_{s s}, D_{p p}$, and $D_{p s}$. On the other hand, the values of $D_{s p}$ are significantly affected by polydispersity. The accuracy of our corrective procedure is very good when $D_{p p} / D_{s s}$ is small $(\sim 0.1)$. This condition is normally satisfied for polydisperse macromolecules in the presence of additives with low molecular weight.

Acknowledgment. The authors are indebted to Prof. John G. Albright for his invaluable assistance with the Gosting diffusiometer and helpful comments on the manuscript. This work was supported by the ACS Petroleum Research Fund (47244-G4) and TCU Research and Creative Activity Funds.

\section{Appendix}

Eigenvector Matrices Used in the Corrective Procedure. For the macromolecule $(p)$-additive $(s)$-solvent system, the eigenvectors of the diffusion-coefficient matrix are obtained by solving the following eigenvalue equation:

$\left[\begin{array}{llllll}D_{11} & D_{12} & D_{13} & \ldots & D_{1} N & D_{1} s \\ D_{21} & D_{22} & D_{23} & \ldots & D_{2} N & D_{2 s} \\ D_{31} & D_{32} & D_{33} & \ldots & D_{3 N} & D_{3 s} \\ \ldots & \ldots & \ldots & \ldots & \ldots & \ldots \\ D_{\mathrm{N} 1} & D_{\mathrm{N} 2} & D_{\mathrm{N} 3} & \ldots & D_{N N} & D_{N s} \\ D_{\mathrm{s} 1} & D_{\mathrm{s} 2} & D_{\mathrm{s} 3} & \ldots & D_{s N} & D_{s s}\end{array}\right] \times$
$\left.\qquad \begin{array}{l}T_{1 k} \\ T_{2 k} \\ T_{3 k} \\ \ldots \\ T_{N k} \\ T_{s k}\end{array}\right]=\Lambda_{k}\left[\begin{array}{l}T_{1 k} \\ T_{2 k} \\ T_{3 k} \\ \ldots \\ T_{N k} \\ T_{s k}\end{array}\right]$ with $k=1,2,3 \ldots, N, s \quad(\mathrm{~A} 1)$

For our corrective procedure, we assume that $D_{i j}=0$ with $i, j$ $=1,2, \ldots, N$ and $i \neq j ; D_{i s}=w_{i} D_{p s}$ with $i=1,2, \ldots, N$; and $D_{s j}=$ $D_{s p}$ with $j=1,2, \ldots, N$.

Equations 27 were obtained by setting $D_{s p}=0$ for $\Gamma_{k}{ }^{\left(\alpha_{p}=0\right)}$, and $D_{p s}=0$ for $\Gamma_{k}{ }^{\left(\alpha_{p}=1\right)}$. The eigenvector matrix used for the $\Gamma_{k}^{\left(\alpha_{p}=0\right)}$ case is

$$
T^{\left(\alpha_{p}=0\right)}=\left[\begin{array}{cccccl}
1 & 0 & 0 & \ldots & 0 & w_{1} D_{\mathrm{ps}} /\left(D_{\mathrm{ss}}-D_{11}\right) \\
0 & 1 & 0 & \ldots & 0 & w_{2} D_{\mathrm{ps}} /\left(D_{\mathrm{ss}}-D_{22}\right) \\
0 & 0 & 1 & \ldots & 0 & w_{3} D_{\mathrm{ps}} /\left(D_{\mathrm{ss}}-D_{33}\right) \\
\ldots & \ldots & \ldots & \ldots & \ldots & \ldots \\
0 & 0 & 0 & \ldots & 1 & w_{\mathrm{N}} D_{\mathrm{ps}} /\left(D_{\mathrm{ss}}-D_{\mathrm{NN}}\right) \\
0 & 0 & 0 & \ldots & 0 & 1
\end{array}\right]
$$

and its inverse is

$$
\left(T^{\left(\alpha_{p}=0\right)}\right)^{-1}=\left[\begin{array}{llllll}
1 & 0 & 0 & \ldots & 0 & -w_{1} D_{\mathrm{ps}} /\left(D_{\mathrm{ss}}-D_{11}\right) \\
0 & 1 & 0 & \ldots & 0 & -w_{2} D_{\mathrm{ps}} /\left(D_{\mathrm{ss}}-D_{22}\right) \\
0 & 0 & 1 & \ldots & 0 & -w_{3} D_{\mathrm{ps}} /\left(D_{\mathrm{ss}}-D_{33}\right) \\
\ldots & \ldots & \ldots & \ldots & \ldots & \ldots \\
0 & 0 & 0 & \ldots & 1 & -w_{\mathrm{N}} D_{\mathrm{ps}} /\left(D_{\mathrm{ss}}-D_{\mathrm{NN}}\right) \\
0 & 0 & 0 & \ldots & 0 & 1
\end{array}\right]
$$

The eigenvector matrix used for the $\Gamma_{k}^{\left(\alpha_{p}=1\right)}$ case is 


$$
\begin{aligned}
& T^{\left(\alpha_{p}=1\right)}=\left[\begin{array}{llllll}
-\left(D_{\mathrm{ss}}-D_{11}\right) D_{s p} & 0 & 0 & \ldots & 0 & 0 \\
0 & -\left(D_{\mathrm{ss}}-D_{22}\right) D_{s p} & 0 & \ldots & 0 & 0 \\
0 & 0 & -\left(D_{\mathrm{ss}}-D_{33}\right) D_{s p} & \ldots & 0 & 0 \\
\ldots & \ldots & \ldots & \ldots & \ldots & \ldots \\
0 & 0 & 0 & \ldots & -\left(D_{\mathrm{ss}}-D_{\mathrm{NN}}\right) D_{s p} & 0 \\
1 & 1 & 1 & \ldots & 1
\end{array}\right]
\end{aligned}
$$

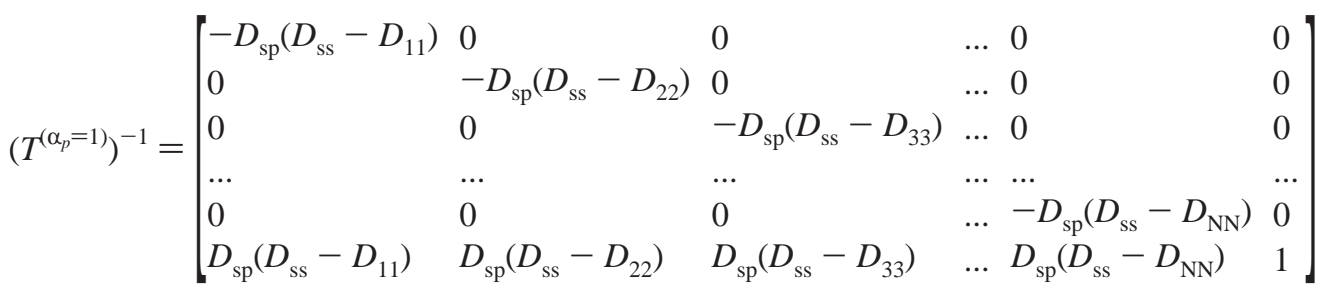

\section{References and Notes}

(1) Cussler, E. L. Diffusion, 2nd ed.; Cambridge University Press: Cambridge, U.K., 1997.

(2) Tyrrell, H. J. V.; Harris, K. R. Diffusion in Liquids; Butterworths: London, 1984

(3) Crank, J. The Mathematics of Diffusion; Oxford at the Clarendon Press: Oxford, 1956.

(4) Sundelöf, L.-O. Ark. Kemi 1965, 25, 1-65.

(5) Cussler, E. L. J. Phys. Chem. 1965, 69, 1144-1148.

(6) Pusey, P. N.; Tough, R. J. A. In Dynamic Light Scattering; Pecora,

R., Ed.; Plenum Press: New York, 1985; pp 85-179.

(7) Schmitz, K. S. Introduction to Dynamic Light Scattering by Macromolecules; Academic Press: San Diego, 1990.

(8) Vergara, A.; Paduano, L.; Vitagliano, V.; Sartorio, R. J. Phys. Chem. $B$ 1999, 103, 8732-8738.

(9) Vergara, A.; Paduano, L.; Vitagliano, V.; Sartorio, R. Phys. Chem. Chem. Phys. 1999, 23, 5377-5383.

(10) Mangiapia, G.; Paduano, L.; Vergara, A.; Sartorio, R. J. Phys. Chem. B 2003, 107, 7216-7224.

(11) Callendar, R.; Leaist, D. G. J. Solution Chem. 2006, 35, 353-379.

(12) Kelly, B.; Leaist, D. G. Phys. Chem. Chem. Phys. 2004, 6, 55235530 .

(13) Creeth, J. M. J. Am. Chem. Soc. 1955, 77, 6428-6440.

(14) Creeth, J. M.; Gosting, L. J. J. Phys. Chem. 1958, 62, 58-65.

(15) Fujita, H.; Gosting, L. J. J. Am. Chem. Soc. 1956, 78, 1099-1106.

(16) Fujita, H.; Gosting, L. J. J. Phys. Chem. 1960, 64, 1256-1263.

(17) Leaist, D. G.; Hao, L. J. Phys. Chem. 1993, 97, 7763-7768.

(18) Annunziata, O.; Buzatu, D.; Albright, J. G. Langmuir 2005, 21, 12085-12089.

(19) Albright, J. G.; Annunziata, O.; Miller, D. G.; Paduano, L.; Pearlstein A. J. J. Am. Chem. Soc. 1999, 121, 3256-3266.

(20) Annunziata, O.; Paduano, L.; Pearlstein, A. J.; Miller, D. G.; Albright, J. G. J. Am. Chem. Soc. 2000, 122, 5916-5928.

(21) Annunziata, O.; Paduano, L.; Pearlstein, A. J.; Miller, D. G.; Albright, J. G. J. Phys. Chem. B 2006, 110, 1405-1415.

(22) Annunziata, O.; Paduano, L.; Albright, J. G. J. Phys. Chem. B 2006, $110,16139-16147$
(23) Annunziata, O.; Paduano, L.; Albright, J. G. J. Phys. Chem. B 2007, $111,10591-10598$

(24) Leaist, D. G. J. Phys. Chem. 1989, 93, 474-479.

(25) Vergara, A.; Paduano, L.; Mangiapia, G.; Sartorio, R. J. Phys. Chem. B 2001, 105, 11044-11051.

(26) Vergara, A.; Paduano, L.; Sartorio, R. Macromolecules 2002, 35, $1389-1398$.

(27) Capuano, F.; Vergara, A.; Paduano, L.; Annunziata, O.; Sartorio, R. J. Phys. Chem. B 2003, 107, 12363-12369.

(28) Vergara, A.; Capuano, F.; Paduano, L.; Sartorio, R. Macromolecules 2006, 39, 4500-4506.

(29) Gosting, L. J. Adv. Protein Chem. 1956, 11, 429-554.

(30) Miller, D. G.; Albright, J. G. In Measurement of the Transport Properties of Fluids: Experimental Thermodynamics; Wakeham, W. A., Nagashima, A., Sengers, J. V., Eds.; Blackwell Scientific Publications: Oxford, 1991; Vol. III, pp 272-294.

(31) Miller, D. G. J. Solution Chem. 1981, 10, 831-845.

(32) Albright, J. G.; Sherrill, B. C. J. Solution Chem. 1979, 8, 201215.

(33) Flory, P. J. Principles of Polymer Chemistry; Cornell University Press: New York, 1953.

(34) Brandrup, J.; Immergut, E. H.; Grulke, E. A. Polymer Handbook, 4th ed.; J. Wiley \& Sons: New York, 1999.

(35) Annunziata, O.; Albright, J. G. Ann. N.Y. Acad. Sci. 2002, 974, 610-616.

(36) Castagnolo, D.; Vergara, A.; Paduano, L.; Sartorio R.; Annunziata, O. Acta Crystallogr. 2002, D58, 1633-1637.

(37) Miller, D. G. J. Phys. Chem. 1988, 92, 4222-4226.

(38) Lomakin, A.; Teplow, D. B.; Benedek, G. B. In Methods in Molecular Biology Amyloid Proteins: Methods and Protocols; Sigurdsson, E. M., Ed.; Humana Press: Totowa, NJ, 2005; Vol. 299, pp 153-174.

(39) Hong, P.-D.; Chou, C.-M.; He, C.-H. Polymer 2001, 42, 61056112.

(40) Kjøniksen, A.-L.; Nyström, B. Macromolecules 1996, 29, 71167123.

(41) Koppel, D. E. J. Chem. Phys. 1972, 57, 4814-4820. 